\title{
Aminopyrine breath test in the prognostic evaluation of patients with cirrhosis
}

\author{
C Merkel, M Bolognesi, S Bellon, S Bianco, B Honisch, H Lampe, P Angeli, A Gatta
}

\begin{abstract}
This prospective study assessed the role of aminopyrine breath test in the prognosis of patients with cirrhosis, and evaluated whether the test provided useful information not included in the Pugh score. During a period of 36 months, 125 patients with biopsy proven liver cirrhosis were included, and followed for up to 48 months (median 17 months). During follow up 43 patients died ( 20 of liver failure). Survival was univariately related to aminopyrine breath test $(\mathrm{p}<0.02)$, Pugh score $(p<0.01)$, presence of ascites $(p<0.01)$, and sex $(p<0.05)$. Using Cox's regression analysis, Pugh score, aminopyrine breath test, and sex, were independent significant predictors of survival. From the Cox's model a prognostic index was computed. According to a receiver operating characteristic curve analysis, the prognostic index predicting death showed an improvement in area under the curve when compared with a prognostic index calculated excluding aminopyrine breath test, but the improvement did not reach statistical significance $(p=0 \cdot 12)$. A similar prognostic index was calculated to predict death from liver failure. Cox's regression analysis selected aminopyrine breath test, Pugh score, and aetiology as the best set of predictor covariates. According to a receiver operating characteristic curve analysis, a prognostic index cut off value of $\mathbf{2 \cdot 6}$ had a $94 \%$ sensitivity and a $88 \%$ specificity. The prognostic index significantly improved prognostic accuracy when compared with a prognostic index calculated from Pugh score and aetiology, but excluding aminopyrine breath test $(p=0.05)$. These data disclose that the aminopyrine breath test offers additional prognostic information to the Pugh score, and it may become a useful tool to better assess the prognosis of patients with cirrhosis.
\end{abstract}

Department of Clinical Medicine, University of Padua, Italy

C Merkel

M Bolognesi

$S$ Bellon

S Bianco

B Honisch

H Lampe*

$P$ Angeli

A Gatta

Correspondence to:

Dorrespondence to:

Dr Carlo Merkel, Istituto di

Clinica Medica II, Policlinico

Clinica Medica II, Policlinico

2, I-35100 Padova, Italy.

Accepted for publication

26 September 1991

^Present address: Department

of Pathology, University o

Düsseldorf, Düsseldorf,

Federal Republic of Germany.

offered to patients with end stage liver disease the prognosis of patients with liver cirrhosis has aroused great interest. From a clinical point of view, the common clinical and biochemical data included in the Child-Turcotte-Pugh classification' ${ }^{1}$ are considered the most useful indicators of prognosis in these patients. ${ }^{2-4}$ In addition, they possess the advantage of easy feasibility and low cost. A more precise definition of prognosis in cirrhosis is desirable, however, and different approaches have been attempted to improve prognostic accuracy..$^{5-10}$

The aminopyrine breath test $"$ is considered useful to evaluate patients with liver disease. ${ }^{1213}$ It is a non-invasive quantitative liver function test, which accurately predicts the outcome of acute liver failure, ${ }^{14}$ of alcoholic hepatitis, ${ }^{15}$ and the risk of surgery in patients with liver disease. ${ }^{16}$ Its role in the prognostic evaluation of patients with cirrhosis is uncertain. Although it is consistently found to be a predictor of survival when considered alone, ${ }^{17-20}$ in two previous studies it has not been shown to significantly improve the prognostic accuracy resulting from the Pugh score. ${ }^{1820}$

The aim of this study is to assess the usefulness of the aminopyrine breath test in improving prognostic accuracy obtained from the Pugh score in patients with cirrhosis of the liver.

\section{Methods}

PATIENTS

During a period of 36 months, all patients with liver cirrhosis, admitted to the Department of Clinical Medicine, University of Padua, who fulfilled the entry criteria were entered into the study. These criteria were (i) presence of biopsy proven cirrhosis, (ii) age less than 75 years, (iii) absence of other diseases having a short prognosis per se, (iv) informed consent and willingness to cooperate in the study. One hundred and twenty five patients were selected from a group of 167 patients with chronic liver disease. Clinical and biochemical data in the 42 excluded patients are reported in Table I. Reasons for exclusion were lack of histological diagnosis in 15 patients (inability to perform the biopsy in 11, refusal in four), extrahepatic malignancies in 10 patients, hepatocellular carcinoma shown in the index or previous admission in seven patients, inability to collaborate in six patients, age over 75 years in four patients. Excluded patients did not differ in any clinical or biochemical variable from the group of 125 included patients (Table I). In these 125 patients reasons for admission were ascites and/or oedema in 69 patients, presence of oesophageal varices without previous bleeding in 20 , previous digestive haemorrhage for which patients were referred for possible treatment in 12 , and acute gastrointestinal bleeding in 19 . Five patients were admitted for unspecific complaints.
TABLE

\begin{tabular}{ll}
\hline Age & $55(43-64)$ \\
Sex $(M / F)$ & $35 / 7$ \\
Aetiology (alcoholic/non-alcoholic) & $29 / 13$ \\
Ascites & 19 \\
Encephalopathy (grade I/II) & $5 / 3$ \\
s-albumin $(g / l)$ & $33(28-39)$ \\
s-bilirubin $(\mu \mathrm{mol} / \mathrm{l})$ & $24(16-45)$ \\
Prothrombin index $(\%)$ & $50(34-80)$
\end{tabular}

Reference values: s-albumin: $35-50 \mathrm{~g} / \mathrm{l}$; s-bilirubin 2-17 $\mu \mathrm{mol} / \mathrm{l}$; interquartile range, when applicable. No significant difference Whitney test, or Fisher's exact test, when applicable). prothrombin index: $80-100 \%$. Data are expressed as median an from the group of 125 included patients in any variable (Mann- 
TABLE II Clinical and biochemical data of the 125 included patients

\begin{tabular}{ll}
\hline Age & $56(45-63)$ \\
Sex $(M / F)$ & $97 / 28$ \\
Aetiology (alcoholic/non-alcoholic) & $94 / 31$ \\
Ascites & 75 \\
Encephalopathy (grade I/II) & $7 / 4$ \\
s-albumin $(g / 1)$ & $33(27-38)$ \\
s-bilirubin $(\mu \mathrm{mol} / \mathrm{l})$ & $28(14-48)$ \\
Prothrombin index $(\%)$ & $54(42-65)$ \\
Pugh score & $8(6-9)$ \\
\hline
\end{tabular}

Reference values: s-albumin: $35^{-}-50 \mathrm{~g} / \mathrm{l}$; s-bilirubin 2-17 $\mu \mathrm{mol} / \mathrm{l}$; prothrombin index: $80-100 \%$. Data are expressed as median and interquartile range, when applicable.

Aetiology of cirrhosis was alcoholic in 94 patients, posthepatitic in 24, cryptogenic in seven. No patient had primary biliary cirrhosis, haemochromatosis, or Wilson's disease.

Medication at the time of the study included cimetidine in five patients, ranitidine in 29 patients, spironolactone in 21 patients, potassium canrenoate in four patients, furosemide in 17 , antibiotics in seven, and digoxin in six patients. A recent history of alcohol consumption was present in most patients with alcoholic cirrhosis (70/94). Clinical and biochemical data in the 125 patients are given in Table II.

\section{STUDY PROTOCOL}

At the time of inclusion in the study, the common clinical and biochemical data included in the Child-Turcotte-Pugh classification were obtained, and the Pugh score' was calculated by summing up the points obtained from the five items in the classification (Table II). The aminopyrine breath test was carried out according to Hepner and Vesell" after an oral dose of $2 \mu \mathrm{Ci}$ of [14-C]-aminopyrine (New England Nuclear, Boston, Mass, USA), and was expressed as per cent of labelled carbon dioxide expired at two hours. Reference range for control subjects in our laboratory is $8-13 \%$. Details of this procedure have been previously described. ${ }^{21}$ No patient experienced complications or side effects related to the test.

Patients were treated according to standard medical practice, and were seen as outpatients every three months and as inpatients when necessary, and were followed for up to 48 months, with a median of 17 months (21 in censored patients). Range of time to death or censoring was $0 \cdot 5-38$ months and $12-48$ months, respectively. Two patients were lost to follow up after three and six months, respectively. Three patients, who died of diseases not related to cirrhosis (myocardial infarction, colonic cancer, lung cancer) were censored at the time of death. All other deaths were considered liver related. Pharmacological treatment included diuretics in the presence of fluid retention, lactulose if encephalopathy was present, blood transfusion, $\mathrm{H}_{2}$ blockers, glypressin, or Blakemore tubing in cases of acute gastrointestinal bleeding. Beta adrenergic blockers, endoscopic sclerotherapy, or portal systemic shunt were offered to prevent rebleeding. Because these treatments have been reported to be substantially equivalent as far as survival is concerned, ${ }^{22}$ the treatment received for prevention of rebleeding was not considered in the analysis.
End point of the study was death by liver related causes. It was classified as caused by liver failure, if progressive impairment of liver function with neurological disturbances occurred, or by gastrointestinal bleeding, if it occurred within 40 days, regardless of the severity of bleeding. This definition of bleeding related death has recently been suggested by a consensus conference. ${ }^{22}$ Hepatorenal syndrome, spontaneous bacterial peritonitis, and other infections were diagnosed according to established criteria. Patients who developed hepatocellular carcinoma during follow up were considered together with the other cirrhotics, and their cause of death was classified according to the clinical presentation. Secondary end point of the study was death from liver failure. To perform this analysis, patients who died of different causes were censored at the time of death.

\section{DATA ANALYSIS}

Statistics are given as medians and interquartile ranges.

To evaluate the prognostic value of the aminopyrine breath test and the results of the conventional investigations, a univariate analysis was performed building Kaplan-Meier plots ${ }^{23}$ for each variable. To verify whether variables were univariately related to survival, curves were compared by the log-rank test, ${ }^{2+}$ or the $t$ test for trend, ${ }^{25}$ when two or three curves had to be compared, respectively.

In order to establish whether the aminopyrine breath test contributed to a better definition of the prognosis in our subjects, after the conventional data had been taken into account, a multiple regression analysis according to the Cox's proportional hazard model $^{26}$ was performed. In a first step the investigated variables were Pugh score, aminopyrine breath test, age, sex, aetiology, history of previous gastrointestinal bleeding, s-creatinine. In a second step, the Pugh score was substituted by the five items that define the score - namely, ascites, encephalopathy, s-albumin, s-bilirubin, and prothrombin index. Variables were first subjected to univariate analysis, then a predictor covariate was added in a stepwise fashion if the $\chi^{2}$ statistics was the greatest, and its $p$ value was less than $0 \cdot 10$. Each chosen covariate was then reconsidered and eliminated if its $\chi^{2}$ value was the smallest, and its $p$ value was higher than $0 \cdot 15$. The procedure continued stepwise until no further covariate could be added or removed according to the above mentioned criteria. To check the proportionality of risk with time in the different classes, the log minus log of cumulative hazard was plotted against time, and showed parallelism between patients with low and high values of selected predictor covariates. To evaluate the contribution provided by each parameter, the coefficient/standard error ratio was computed, and the models obtained including and excluding these variables were compared by the Wald test. ${ }^{27}$

To assess the validity of the models proposed, a split sample procedure was used. Patients were randomly divided into a training sample which 
comprised approximately $60 \%$ of the patients, and a test sample which included the remainders. Validation was performed according to Schlichting et al. ${ }^{6}$

The clinical usefulness of the aminopyrine breath test was assessed by deriving prognostic indices ${ }^{27}$ which included or excluded the test. Sensitivity and specificity of prognostic indices predicting death or death from liver failure within 18 months were computed according to standard formulae, and receiver operating characteristic curves were drawn. Areas under the curve and standard errors of areas under curve were calculated and compared according to Hanley and McNeil. ${ }^{28} 29$ Because the null hypothesis predicts that no improvement in areas under curve exists after adding the aminopyrine breath test, a one sided significance test was used. All statistical analysis was performed using the BMDP statistical package. ${ }^{30}$

\section{Results}

FACTORS PREDICTING DEATH FROM LIVER RELATED CAUSES

During follow up 43 patients died: 20 of liver failure, 15 of gastrointestinal bleeding, three of infections, two of hepatorenal syndrome, and three of diseases not related to cirrhosis.

According to univariate analysis survival was significantly related to the aminopyrine breath test $(\mathrm{p}<0.02)$, Pugh score $(\mathrm{p}<0.01)$, s-albumin $(\mathrm{p}<0.01)$, s-bilirubin $(\mathrm{p}<0.05)$, prothrombin index $(p<0.01)$, presence of ascites $(p<0.01)$, presence of encephalopathy $(\mathrm{p}<0.01)$, and sex $(p<0.05)$. It was not related to the aetiology of cirrhosis, or to a history of gastrointestinal bleeding.

Cox's multiple regression analysis selected Pugh score, the aminopyrine breath test, and sex as the best set of independent and significant predictors of survival. The statistical parameters of the model are given in Table III. A prognostic index predicting death (PI-d) was derived as:

PI-d $=0 \cdot 299 \times($ Pugh score $)-0 \cdot 25 \times$

(aminopyrine breath test) $-0.955 \times(\mathrm{sex})$,

attributing a value of 1 for male sex and two for female sex. Median prognostic index predicting death in the whole series was 0.30 (interquartile range: $-0.52+1 \cdot 24)$. The relationships between PI-d and survival are illustrated in Figure 1.

When the values of the five items included in

TABLE III Statistical parameters of Cox's models predicting death from liver-related causes or death from liver failure in 125 patients with cirrhosis

\begin{tabular}{lccrc}
\hline & & \multicolumn{2}{c}{ Improvement } \\
\cline { 3 - 5 } Variable & Coeff. & Coeff./SE & $\chi^{2}$ & \multicolumn{1}{c}{$p$} \\
\hline Death from liver-related causes & & & \\
Pugh score & 0.299 & 3.430 & 22.51 & $<0.001$ \\
ABT & -0.250 & -2.505 & 6.53 & 0.01 \\
Sex & -0.955 & -1.807 & 4.19 & 0.04 \\
Death from liver failure & & & \\
ABT & -0.829 & -3.557 & 33.67 & $<0.001$ \\
Pugh score & 0.609 & 4.051 & 15.52 & $<0.001$ \\
Aetiology & -1.203 & -1.845 & 4.21 & 0.04 \\
\hline \multirow{2}{*}{ Male $=1$; Female $=2}$. & & & \\
†Alcoholic aetiology $=1$; non-alcoholic aetiology $=2$. &
\end{tabular}
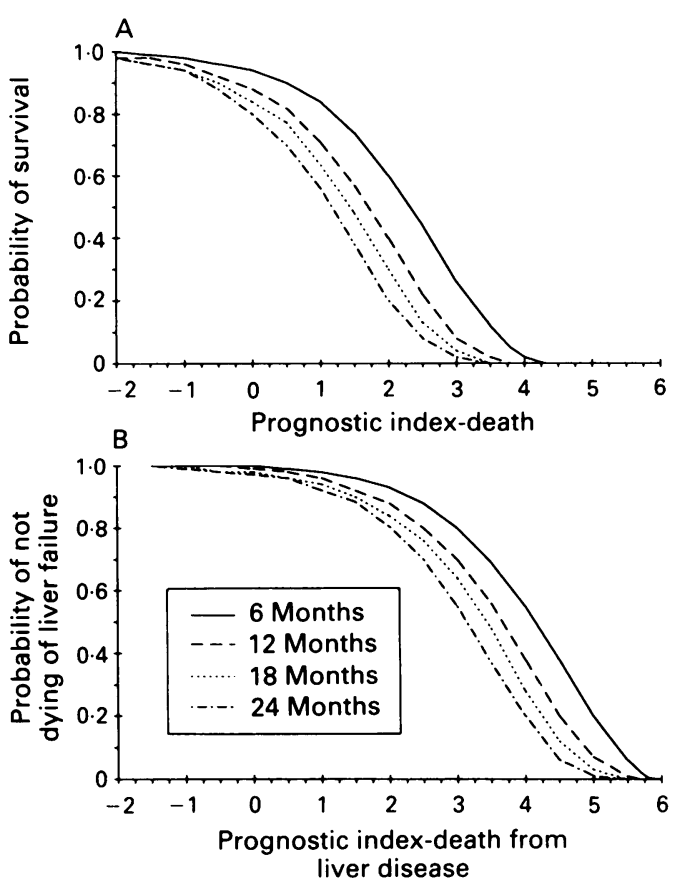

Figure 1: Relationships between the prognostic index predicting death $(P I-d)$ and the probability of survival at different time intervals $(A)$, and relationships between the prognostic index predicting death from liver failure (PI-1f) and the risk of death from liver failure $(B)$.

the Pugh score were substituted to the Pugh score as candidate predictor covariates in Cox's analysis, aminopyrine breath test encephalopathy, ascites, sex, and s-bilirubin were selected by the computer procedure. Global goodness of fit was not significantly different, and aminopyrine breath test regression coefficient was nearly identical. The statistical parameters of this model are given in Table IV.

FACTORS PREDICTING DEATH FROM LIVER FAILURE The risk of death from liver failure was univariately related to the aminopyrine breath test $(\mathrm{p}<0.01)$, Pugh score $(\mathrm{p}<0.01)$, s-albumin $(\mathrm{p}<0.01)$, s-bilirubin $(\mathrm{p}<0.01)$, prothrombin index $(p<0.01)$, presence of ascites $(p<0.01)$, or encephalopathy $(p<0.01)$. It was not univariately related to sex, aetiology of cirrhosis, or history of gastrointestinal bleeding.

TABLE IV Statistical parameters of Cox's models predicting death from liver-related causes or death from liver failure in 125 patients with cirrhosis, when the five variables included in the Pugh score were substitued to the score

\begin{tabular}{lcrrr}
\hline & & & \multicolumn{2}{c}{ Improvement } \\
\cline { 5 - 6 } Variable & Coeff. & Coeff./SE & \multicolumn{1}{c}{$\chi^{:}$} & \multicolumn{1}{c}{$p$} \\
\hline Death from liver-related causes & & & \\
ABT & -0.250 & -2.465 & 16.73 & $<0.001$ \\
Encephalopathy & 0.725 & 2.300 & 9.22 & 0.002 \\
Ascitest & -0.806 & -2.133 & 6.31 & 0.01 \\
Sex & -1.048 & -1.969 & 5.97 & 0.02 \\
s-bilirubin & 0.007 & 1.991 & 3.23 & 0.07 \\
Death from liver failure & & & \\
ABT & -0.945 & -3.992 & 33.67 & $<0.001$ \\
Ascitest & -2.468 & -2.365 & 13.06 & $<0.001$ \\
s-bilirubin & 0.014 & 3.203 & 8.02 & 0.005 \\
\hline
\end{tabular}

\#Without encephalopathy $=0$; with encephalopathy $=1$. + Without ascites $=0$; with ascites easy to control $=1$; with resistant ascites $=2$.

$\ddagger$ Male $=1 ;$ female $=2$. 

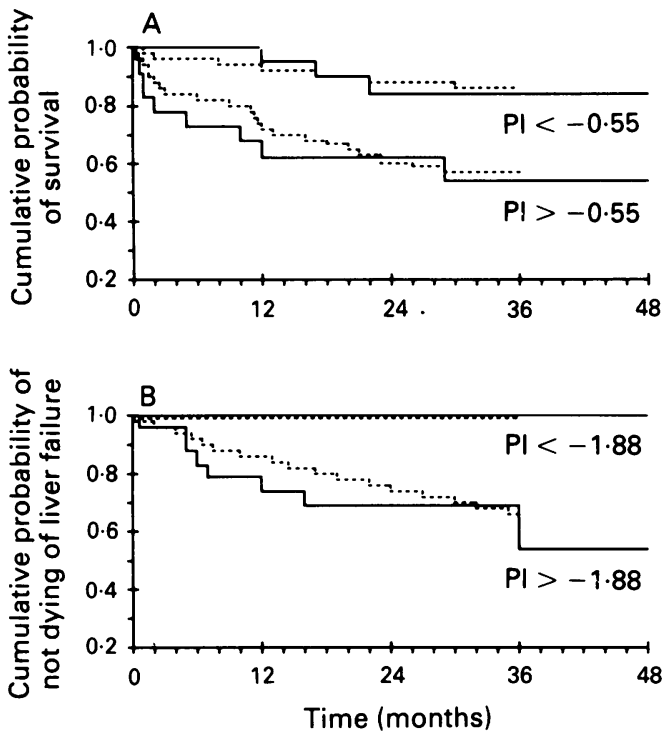

Figure 2: Validation of Cox's models. (A) cumulative probability of survival in the patients of the test set divided according to the values of prognostic index predicting death (solid lines), and probability of survival calculated from the training set according to the Cox's model (dotted lines). (B) cumulative probability of not dying of liver failure in the patients of the test set divided according to the levels of prognostic index predicting death from liver failure (solid lines), and probability of not dying of liver failure calculated from the training set according to the Cox's model (dotted lines).

Applying Cox's regression analysis, the aminopyrine breath test, the Pugh score, and aetiology were the best set of covariates significantly predicting death from liver failure. The statistical parameters of this model are given in Table III. From the model a prognostic index predicting death from liver failure (PI-1f) was computed as:

PI-If $=0.609 \times($ Pugh score $)-0 \cdot 829 \times$ (aminopyrine breath test) $-1 \cdot 203 \times($ aetiology), attributing a value of 1 for alcoholic aetiology, and 2 for non-alcoholic aetiology. Median prognostic index predicting death from liver failure in the whole series was 0.41 (interquartile range: $-1 \cdot 64+2 \cdot 16)$. The relationships between PI-lf and risk of death from liver failure is illustrated in Figure 1.

When Cox's regression analysis was performed including the variables which constitute the Pugh score, selected predictor covariates were the aminopyrine breath test, ascites, and s-bilirubin. Overall goodness of fit was not significantly different from that of the model containing Pugh score. The aminopyrine breath test regression coefficient was similar. Statistical parameters of this model are given in Table IV.

\section{VALIDITY OF THE MODELS}

This was assessed using a split sample technique. Prognostic index predicting death and prognostic index predicting death from liver failure were recalculated in a training set of approximately $60 \%$ of randomly selected patients. Individual prognostic indices were calculated for the test set comprising the remainders. Regression coefficients in the training set were very similar to those of the complete set of 125 patients. The test set was divided into two groups based upon prognostic indexes above or below the median value, and mean estimated survival function for the patients in the two classes were computed. These were compared with the observed outcome in the same patients calculated according to Kaplan-Meier plots. No significant difference between observed and expected mortality, or mortality from liver failure, was found in patients with different levels of prognostic indexes (Fig 2).

\section{CLINICAL USEFULNESS OF PROGNOSTIC INDEXES}

To evaluate the clinical usefulness of the addition of the aminopyrine breath test to the common data in the prediction of death, prognostic index predicting death (which comprises Pugh score, the aminopyrine breath test and sex) was compared with a prognostic index computed only from Pugh score and sex. Sensitivity and specificity in predicting death within 18 months were calculated in eight equal intervals of prognostic indexes, and receiver operating characteristic curves were drawn. The best discriminant point of prognostic index predicting death was

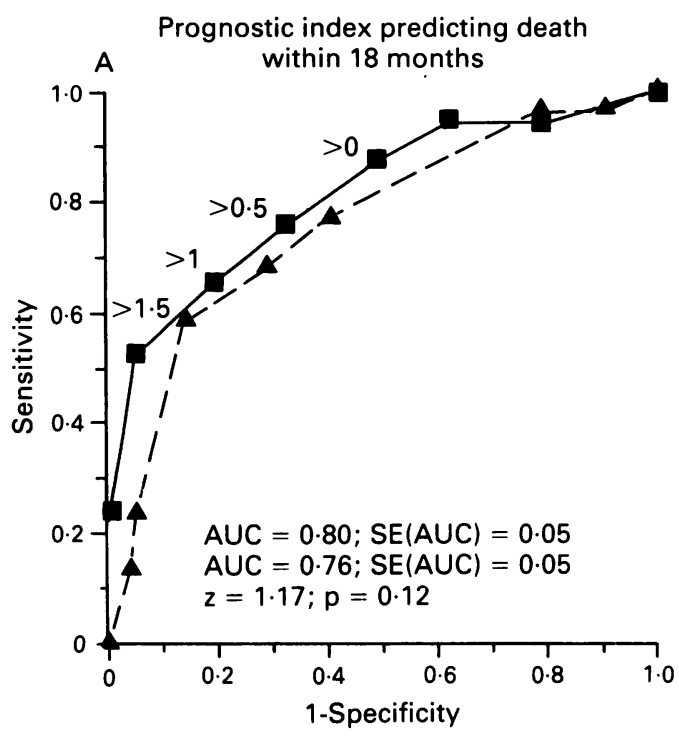

Prognostic index predicting death from liver B failure within 18 months

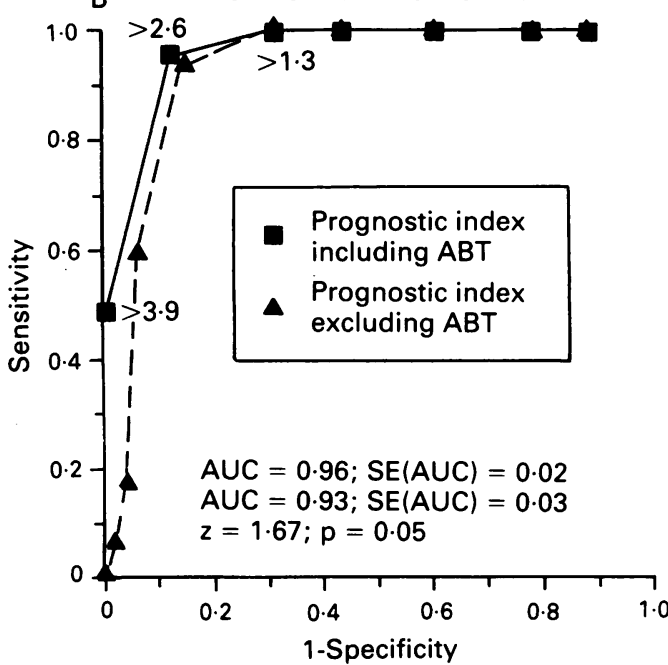

Figure 3: Receiver operating characteristic curves of the prediction of death (upper panel) or of death from liver failure (lower panel) according to prognostic indices computed including $A B T$ (solid lines) or excluding $A B T$ (dashed lines). 
0.5 ; which had a $75 \%$ sensitivity and a $67 \%$ specificity in predicting death within 18 months. The receiver operating characteristic curve calculated from prognostic index predicting death was always nearer to the upper left corner than that calculated without the aminopyrine breath test (Fig 3). Areas under curve calculated from prognostic index predicting death $(0 \cdot 80$; $\mathrm{SE}(\mathrm{AUC})=0.05)$ was larger than that calculated from a prognostic index excluding the aminopyrine breath test $(0.76 ;$ SE $($ AUC $)=0.05)$, but the difference did not reach statistical significance $(z=1 \cdot 17 ; p=0 \cdot 12$; one-sided test).

A similar analysis was performed to assess the clinical usefulness of prognostic index predicting death from liver failure. Prognostic index predicting death from liver failure (obtained from the aminopyrine breath test, the Pugh score, and aetiology) was compared with a prognostic index calculated excluding aminopyrine breath test, and receiver operating characteristic curves were drawn. The best discriminant point for prognostic index predicting death from liver failure was $2 \cdot 6$, which had a sensitivity of $94 \%$ and a specificity of $88 \%$ in predicting death from liver failure within 18 months (Fig 3). The receiver operating characteristic curve drawn according to prognostic index predicting death from liver failure always depicted a better performance than that of the prognostic index obtained excluding aminopyrine breath test. Areas under curve was also significantly larger $(\mathrm{AUC}=0.96 \mathrm{v}$ 0.93: $\mathrm{SE}(\mathrm{AUC})=0.02 v 0.03 ; \mathrm{z}=1.67 ; \mathrm{p}=0.05$, one-sided test).

\section{Discussion}

In the current study we have provided evidence that aminopyrine breath test is a strong predictor of survival in patients with cirrhosis, and that it adds information not available from the common clinical and biochemical data included in the Pugh score in the assessment of the risk of death from liver failure.

The aminopyrine breath test has already been shown to be useful in conditions of acute liver damage, such as alcoholic hepatitis, ${ }^{15}$ or fulminant hepatic failure. ${ }^{14}$ In numerous series of patients with cirrhosis it was also reported to have a strong predictor effect when considered alone. ${ }^{17-20}$ Only two studies ${ }^{18} 20$ have examined the possibility that the aminopyrine breath test could improve prognostic ability derived from the Pugh score in patients with cirrhosis, and both failed to show significant effects. Villeneuve et $a l,{ }^{18}$ analysing a group of 187 patients with cirrhosis followed for a mean of 28 months, observed that it was not possible to include the aminopyrine breath test in the set of selected covariates in Cox's model predicting death, which comprised most of the data used to define the Pugh score. They concluded that the aminopyrine breath test had no additional prognostic usefulness, once the data included in the Pugh score were considered. Adler et $a l^{20}$ recently developed a stepwise logistic regression to identify cirrhotic patients who were going to die within one year. They observed that the association of the aminopyrine breath test and the presence of ascites was the best set of covariates predicting death, superior to the other common clinical and biochemical data. When the prognostic index derived from the aminopyrine breath test and ascites was compared with the Pugh score, however, the difference in effectiveness was weakly in favour of the aminopyrine breath test and ascites, and it did not reach statistical significance.

At variance with previous reports, the aminopyrine breath test was an important prognostic index in our series. In fact, mortality from liver related causes was best predicted by a prognostic index which contained the Pugh score, the aminopyrine breath test, and sex, and the aminopyrine breath test significantly improved the efficiency of the regression, as assessed by the Wald test. ${ }^{27}$

From a practical point of view, however, one might wonder whether the improvement in the goodness of fit of the regression produced an improvement in the accuracy of the discrimination of patients who will eventually die from those who will survive. The receiver operating characteristic curve analysis showed that sensitivity and specificity of the prognostic index predicting death, containing the aminopyrine breath test, the Pugh score, and sex, were always better than those of a prognostic index derived only from Pugh score and sex. The difference in efficiency did not reach statistical significance, however, probably because of the insufficient sample size, and the relative magnitude of the standard errors. A further observation is that this type of analysis cannot take into account the different times to death, at variance with the Cox's regression model. Therefore a part of information is lost in the receiver operating characteristic curve analysis.

As the previous analysis was not able to show the clinical usefulness of the aminopyrine breath test in predicting liver related death, a secondary analysis was carried out considering only deaths from liver failure, which are expected to be more strictly linked to liver function derangement. This regression equation fitted the data better than that of overall mortality, as shown by the global $\chi^{2}$ values. In this regression the aminopyrine breath test and the Pugh score were still the main prognostic indicators. The third indicator, much less important according to the coefficient/standard error ratio, was aetiology, while in Cox's model predicting overall mortality it was sex. Because alcoholic aetiology and male sex were strictly associated in our series $\left(\chi^{2}=\right.$ $5 \cdot 15 ; p<0.025)$, it is likely that the difference in the third parameter of the regression is expression of the same phenomenon. ${ }^{27}$

In this setting the aminopyrine breath test was the first covariate to enter the regression - that is, the more strictly associated to the risk of death from liver failure - and the weight of the aminopyrine breath test in the overall regression was only marginally less than that of the Pugh score, as assessed by the coefficient/standard error ratio. The prognostic index predicting death from liver failure (obtained from the aminopyrine breath test, the Pugh score, and aetiology) was reasonably stable, when recalculated on $60 \%$ of the data, and proved valid in the assessment of the remaining patients. The 
receiver operating characteristic curve analysis showed that a prognostic index predicting death from liver failure value of 2.6 had a sensitivity of $94 \%$ and a specificity of $88 \%$ in predicting death from liver failure within 18 months. The area under curve of the receiver operating characteristic curve calculated from the complete set of covariates was significantly larger than that of a prognostic index which excluded the aminopyrine breath test. This also implies that from a practical point of view the addition of the aminopyrine breath test to the common data improved efficiency in predicting death from liver failure.

The results of the present study are at variance with those of Villeneuve et $a l,{ }^{18}$ who performed a study very similar to ours. The reason for this difference is not evident. It may be caused by, at least in part, to the different populations studied, as our study mostly comprised patients with alcoholic cirrhosis, and none with primary biliary cirrhosis, while in the Canadian study only $55 \%$ had alcoholic cirrhosis, and $10 \%$ had primary biliary cirrhosis. As it was shown that the aminopyrine breath test is poorly associated to the severity of cholestatic conditions, ${ }^{31}$ the presence of cases with primary biliary cirrhosis in the Canadian study may have contributed to decrease the overall efficiency of the aminopyrine breath test in the whole series. Furthermore, a much larger proportion of patients with alcoholic cirrhosis had a history of recent alcohol consumption in our series, and this may have improved prognostic efficiency, as the aminopyrine breath test was found particularly effective in evaluating the severity of acute alcoholic damage. ${ }^{15}$

The pathophysiological mechanisms through which the aminopyrine breath test appeared to be superior to the Pugh score are entirely speculative, but may include the following: (i) there may be substantial variations in the scoring according to Pugh, because two variables are qualitative $^{2}$; (ii) s-albumin, s-bilirubin, and prothrombin index may change irrespectively of liver function impairment because of different pathophysiological mechanisms ${ }^{13}$; (iii) removal rate of aminopyrine represents an estimate of the ability of the liver in removing the drug, and should be more strictly related to the severity of liver disease than the measurement of a concentration in blood of a substance metabolised by the liver. ${ }^{32}$

In conclusion, the aminopyrine breath test significantly improved the prognostic ability of the Pugh score in the prognosis of death from liver failure in patients with cirrhosis. Should these data be confirmed in other studies in different settings, the aminopyrine breath test could prove to be a useful clinical tool to routinely evaluate the prognosis of patients with cirrhosis in addition to common clinical and biochemical data, because it is non-invasive, safe, and has an acceptable cost.

The authors are indebted to Dr Franco Noventa for help in the statistical analysis. The authors wish also to thank the staff of the Department of Clinical Medicine, University of Padua, for the care of investigated patients.

The study was supported in part by grants from the Italian Ministery of University and Scientific Research (National project 'Liver cirrhosis').
1 Pugh RNH, Murray-Lyon IM, Dawson JL, Pietroni MC, Williams R. Transection of the oesophagus for blecding oesophageal varices. BrF Surg 1973; 60: 646-9.

2 Conn HO. A peek at the Child-Turcotte classification. Hepatology 1981; 1: 673-6.

3 Christensen E, Schlichting P, Fauerholdt L, Gluud C, Andersen PK, Juhl E, et al. Prognostic value of Child-Turcotte criteria in medically treated cirrhosis. Hepatology 1984; 4: $430-5$.

4 Infante-Rivard C, Esuada S, Villeneuve JP. Clinical and statistical validity of conventional prognostic factors in predicting short-term survival among cirrhotics. Hepatology 1987; 7: 660-4

5 Orrego H, Israel Y, Blake JE, Medline A. Assessments of prognostic factors in alcoholic liver disease: towards a global quantitative expression of severity. Hepatology 1983; 3: 896905.

6 Schlichting P, Christensen E, Andersen PK, Fauerholdt L, Juhl E, Poulsen $\mathrm{H}$, et al. Prognostic factors in cirrhosis identified by Cox's regression model. Hepatology 1983; 3: 889-95.

7 Pignon JP, Poynard T, Naveau S, Marteau P, Zourabichvili $\mathrm{O}$, Chaput JC. Analyse multidimensionnelle selon le modéle de Cox de la survie de patients atteints de cirrhose alcolique. Gastroenterol Clin Biol 1986; 10: 461-7.

8 D'Amico G, Morabito A, Pagliaro L, Marubini E, and the Liver Study Group of 'V Cervello' Hospital. Survival and prognostic indicators in compensated and decompensated cirrhosis. Dig Dis Sci 1986; 31: 468-75.

9 Rossi L, Milani A, Marra L, Siciliano M. Grading scores and survivorship functions in liver cirrhosis: a comparative statistical analysis of various predictive models. Hepatogastroenterology 1986; 33: 240-3.

10 Merkel C, Bolognesi M, Finucci GF, Angeli P, Caregaro L, Rondana $M$, et al. Indocyanine green intrinsic hepatic clearance as a prognostic index of survival in patients with cirrhosis. F Hepatol 1989; 9: 16-22.

11 Hepner GW, Vesell ES. Quantitative assessments of hepatic function by breath analysis after oral administration of 14-C aminopyrine. Ann Intern Med 1975; 83: 632-8.

12 Hoffmann AF. The aminopyrine demethylation breath tes and the serum bile acid levels: nominated but not yet elected to join the common liver tests. Hepatology 1982; 2: 512-7.

13 Bircher J. Quantitative assessment of deranged hepatic function: a missed opportunity? Semin Liver Dis 1983; 3 275-84.

14 Saunders JB, Wright N, Lewis KO. Predicting outcome of paracetamol poisoning by using 14-C-aminopyrine breath test. $B M F$ 1980; 280: 279-80.

15 Schneider JF, Baker AL, Haines NW, Hatfield G, Boyer JL. Aminopyrine N-demethylation: a prognostic test of liver function in patients with alcoholic liver disease. Gastroenterology 1980; 79: 1145-50.

16 Gill RA, Goodman MW, Golfus GR. Aminopyrine breath test predicts surgical risk for patients with liver disease. Ann Surg 1983; 198: 701-4.

17 Henry DA, Kitchingman G, Langman MJS. [14-C] aminopyrine breath analysis and conventional biochemical tests as predictors of survival in cirrhosis. Dig Dis Sci 1985; 30 813-8.

18 Villeneuve JP, Infante-Rivard $\mathrm{C}$, Ampelas $\mathrm{M}$, PomierLayrargues G, Huet PM, Marleau D. Prognostic value of the aminopyrine breath test in cirrhotic patients. Hepatology 1986; 6: 928-31.

19 Rasmussen B, Dossing M, Poulsen HE, Tygstrup N. Antipyrine, aminopyrine and s-bilirubin as prognostic indices of five years survival in liver cirrhosis [Abstract] f Hepatol 1987; 5: S189.

20 Adler M, Van Laethem J, Gilbert A, Gelin M, Burgeois N, Vereerstraeten $\mathrm{P}$, et al. Factors influencing survival at one year in patients with nonbiliary hepatic parenchymal cirrhosis. Dig Dis Sci 1990; 35: 1-5

21 Merkel C, Sacerdoti D, Finucci GF, Zuin R, Bazzerla G, Bolognesi $M$, et al. Effect of nadolol on liver haemodynamics and function in patients with cirrhosis. $\mathrm{Br} \mathcal{F}$ Clin Pharmacol 1986; $21: 713-9$.

$\angle L$ Burroughs AK. Methodology and reviews of clmical trials in portal hypertension. Amsterdam: Excerpta Medica, 1987.

23 Kaplan EL, Meier P. Nonparametric estimation from incomplete observations. $\mathcal{F}$ Am Statist Assoc 1958; 53: 45781 .

24 Mantel N, Haenzel W. Statistical aspects of the analysis of data from retrospective studies of diseases. 7 Nat Cancer Inst 1959; 22: 719-48.

25 Peto R, Pike MC, Armitage P, Breslow NE, Cox DR, Howard $\mathrm{SV}$, et al. Design and analysis of randomized clinical trials requiring prolonged observation of each patient. Analysis and examples. $B r \mathcal{F}$ Cancer 1977; 35: 1-39.

26 Cox DR. Regression models and life-tables. $f R$ Stattst Soc 1972; 34: 187-220.

27 Christensen E. Multivariate survival analysis using Cox's regression model. Hepatologv 1987; 7: 13+3-58. 
28 Hanley JA, McNeil BJ. The meaning and use of the area under a receiver operating characteristic (ROC) curve. Radiology 1982; 143: 29-36.

29 Hanley JA, McNeil BJ. A method of comparing the areas under receiver operating characteristic curves derived from the same cases. Radiology 1983; 148: 839-43.

30 Dixon WJ, Brown MB, Engelman L, Frane JW, Hill MA,
Jenrich RI, et al. BMDP statistical software. Berkeley: University of California Press, 1983.

31 Baker AL, Krager PS, Kotake AN, Schoeller DA. The aminopyrine breath test does not correlate with histologic disease severity in patients with cholestasis. Hepatolog 1987; 7: 46+7.

32 Branch RA. Drugs as indicators of hepatic function. Hepatologv 1982; 2: 97-105. 